\title{
Classificação Internacional de Funcionalidade e In- capacidade e Saúde (CIF): processo de elaboração e debate sobre a questão da incapacidade
}

\section{The International Classification of Functioning, Disability and Health (ICF): elaboration process and debate about the disability question}

\author{
Elisabete Ferreira Mângia', Melissa Tieko Muramoto ${ }^{2}$, \\ Selma Lancman ${ }^{1}$
}

\begin{abstract}
MÂNGIA, E. F.; MURAMOTO, M. T.; LANCMAN, S. Classificação Internacional de Funcionalidade e Incapacidade e Saúde (CIF): processo de elaboração e debate sobre a questão da incapacidade. Rev. Ter. Ocup. Univ. São Paulo, v. 19, n. 2, p. 121-130, maio/ago. 2008.
\end{abstract}

\begin{abstract}
RESUMO: O problema da incapacidade tem relevância social e econômica para a maior parte dos países e proporções dramáticas se considerarmos a avaliação sobre a existência de 600 milhões de pessoas incapacitadas no mundo. A Organização Mundial da Saúde (OMS) tem sistematicamente alertado os países sobre a insuficiência de programas de promoção das pessoas com incapacidade e destaca a necessidade de se compreender esse problema como questão de responsabilidade social, especialmente no que diz respeito à garantia dos direitos das pessoas com incapacidades. Nesse contexto, este artigo tem como objetivo apresentar a Classificação Internacional de Funcionalidade, Incapacidade e Saúde - CIF - e algumas discussões subjacentes a ela, tais como: a construção social da incapacidade; o processo de transição entre a CIDID - Classificação Internacional das Deficiências, Incapacidades e Desvantagens - e a CIF; sua base conceitual e algumas considerações teóricas sobre a construção do instrumento. Para isso, explora em especial três artigos, cuja leitura recomendamos: "La seconda classificazione internazionale di defcit, disabilitá e handicap dell'pms: confronto e integrazione di modelli di disabilitá", organizado por Barbara D'Avanzo (2000), "Models of disablement, universalism and international classification of impairments, disabilities and handcaps", de Bickenbach et al. (1999) e "Desmystifying disability: a review of the International Classification of Functioning, Disability and Health", de Rob Imrie.
\end{abstract}

DESCRITORES: Classificação Internacional de Funcionalidade, Incapacidade e Saúde. Responsabilidade social. Defesa das pessoas com deficiência/legislação \& jurisprudência.

\footnotetext{
1. Profa. Dra. do Curso de Terapia Ocupacional Departamento de Fisioterapia, Fonoaudiologia e Terapia Ocupacional da Faculdade de Medicina da Universidade de São Paulo - FMUSP.

2. Mestre em Ciências da Reabilitação pela Faculdade de Medicina da Universidade de São Paulo. Terapeuta Ocupacional do Departamento de Fisioterapia, Fonoaudiologia e Terapia Ocupacional da FMUSP.

Endereço para correspondência: Departamento de Fisioterapia, Fonoaudiologia e Terapia Ocupacional da FMUSP. Rua Cipotânea, 51, Cidade Universitária. São Paulo, SP. CEP: 05360-160.
} 
MÂNGIA, E. F. et al. Classificação Internacional. CIF. Rev. Ter. Ocup. Univ. São Paulo, v. 19, n. 2, p. 121-130, maio/ago. 2008.

$\mathrm{O}$ problema da incapacidade tem relevância social e econômica para a maior parte dos países e proporções dramáticas se considerarmos a avaliação sobre a existência de 600 milhões de pessoas incapacitadas no planeta. Os conflitos armados, todas as modalidades de violências e os acidentes de trabalho e de transito tem contribuído para esse quadro. Alem disso, aumento da esperança de vida e o perfil de morbidade que revela a presença de doenças incapacitantes em vários países também são alguns dos fatores causais das incapacidades. As pessoas incapacitadas constituem $10 \%$ da população geral, porém estima-se sua repercussão sobre $25 \%$ da população total, pois a incapacidade afeta não só a pessoa que a sofre como também aqueles que dela cuidam e/ou dependem, seus familiares, a comunidade e quem quer que apóie o desenvolvimento da comunidade (OMS, 2006).

Essa problemática também não tem sido devidamente enfrentada pelas políticas públicas. O quadro atual não aponta para uma tendência de redução da produção social de incapacidade é faz-se necessário que essa discussão faça parte da agenda política dos governos, que deveriam ampliar os recursos e as estratégias para seu enfrentamento (SARACENO, 1998; OMS, 2006).

Apesar da diversidade de dados e metodologias empregados, estudos desenvolvidos em países da América Latina e do Caribe estimam a existência de 60 milhões de pessoas incapacitadas, das quais, aproximadamente $25 \%$ são crianças e adolescentes. Destacamos que $2 \%$ a $3 \%$ dos recém-nascidos apresentam graves deficiências e $6 \%$ a $8 \%$ da população infantil são de alto risco. A questão das incapacidades atinge todas as faixas etárias e metade das vítimas de acidentes de trânsito são jovens entre $15 \mathrm{e}$ 34 anos. Outro aspecto importante são as estratégias de enfrentamento do problema, uma vez que somente $2 \%$ a $3 \%$ da população com incapacidade têm acesso a programas e serviços de reabilitação. Da população infantil incapacitada, só 20 a 30\% freqüentam escolas e só $5 \%$ terminam o curso primário. As meninas incapacitadas são sujeitas a discriminação ainda maior. As crianças com incapacidade sofrem mais violência e maus-tratos do que qualquer outra criança e freqüentemente são institucionalizadas (OMS, 2006).

Essas pessoas encontram obstáculos com relação ao gozo dos seus direitos humanos e liberdades fundamentais, tais como, acesso aos serviços de saúde e reabilitação, equipamentos urbanos, locomoção e transporte e liberdade de movimento em edifícios públicos; falta de oportunidades profissionais; a exclusão dessas pessoas nos sistemas educacionais; a participação em experimentos médicos ou científicos sem seu consentimento informado. As restrições à participação social incluemfalta de instrumentos de apoio para que as pessoas com incapacidades possam votar e participar de várias esferas da vida social.

Outro aspecto importante é a falta de acesso ou de preparo das instituições de saúde pública para que essas pessoas sejam atendidas, o que pode prejudicar sua saúde física ou mental e outros direitos humanos básicos, com conseqüências muitas vezes irreparáveis (OMS, 2006).

É necessário certo grau de saúde física e mental para poder assegurar o exercício dos direitos e liberdades fundamentais que assegurem a participação na vida civil, social, política e econômica de um Estado, O exercício dos direitos e liberdades humanas mencionadas também é essencial para que as pessoas incapacitadas possam desfrutar de verdadeiro bem-estar físico e mental (OMS, 2001, 2006).

A Organização Mundial da Saúde (OMS) tem sistematicamente alertado os países sobre a insuficiência de programas de promoção das pessoas com incapacidade. Na Assembléia Mundial da Saúde realizada no ano de 2005 a OMS lançou um documento que estabelece que é necessário entender o problema da incapacidade como uma responsabilidade social comum e não individual e alerta para a necessidade de promover e proteger os direitos humanos das pessoas incapacitadas (OMS, 2006).

Nesse contexto se inclui a necessidade de retomar a discussão sobre a atual Classificação Internacional de Funcionalidade, Incapacidade e Saúde (CIF) lançada pela Organização Mundial de Saúde em 2001 e que ainda é pouco conhecida, divulgada, debatida e utilizada em nosso país (OMS, 2003).

Neste artigo buscamos divulgar, em nosso idioma, contribuições já publicadas, de autores que se dedicaram ao estudo do processo que levou à proposição da nova classificação, com especial atenção à discussão teóricopolítica subjacente a ela.

A nova classificação busca superar o modelo biomédico e a tendência dos modelos tradicionais de reabilitação, de considerar a questão da incapacidade de forma redutiva e centrada na pessoa e, mais especificamente, em sua estrutura anátomo-fisiológica ou psicológica. Essa redução, muito debatida na contemporaneidade, produz e alimenta um conjunto de processos discriminatórios que invalidam a diferença e a diversidade humana. Essa cultura está apoiada num modelo normativo que não tem sido colocado em questão nem é considerado em suas dimensões históricas e sociais (SARACENO, 1998).

As idéias aqui apresentadas foram inspiradas pelo debate crescente na literatura e em especial em dois artigos sobre o tema : "La seconda classificazione internazionale di defcit, 
disabilitá e handicap dell'pms: confronto e integrazione di modelli di disabilitá, organizado por D'Avanzo (2000) e "Models of disablement, universalism and international classification of impairments, disabilities and handcaps de Bickenbach et al. (1999), cuja leitura recomendamos aos interessados no aprofundamento desta temática.

È nosso interesse apresentar: o processo de elaboração crítica que resultou na CIF, suas características e impasses atuais, além de recuperar alguns elementos críticos e polêmicos sobre o debate em torno da produção social da incapacidade.

\section{A incapacidade como construção social}

Partindo da compreensão sócio-política do problema, a desabilidade ou incapacidade física ou mental, não pode ser compreendida como atributo de uma pessoa, mas como uma configuração complexa que envolve a relação entre pessoas e seu contexto social. Assim, a incapacidade/desabilidade não pode ser reduzida à limitação decorrente do déficit ou da deficiência de que cada sujeito seria supostamente portador e que determinaria alguma disfunção em sua vida social. Essa perspectiva se contrapõe ao modelo médico que compreende o dano ou déficit em seu aspecto anatômico funcional e como atributo do indivíduo (BICKENBACH et al., 1999).

D'Avanzo (2000) identifica duas principais matrizes teóricas nos estudos críticos sobre a incapacidade. De um lado, a perspectiva funcionalista, mais presente nos estudos norte-americanos, e de outro a materialista, localizada na tradição britânica.

A corrente funcionalista constrói argumentos em torno ao conceito de estigma, da exclusão dos incapacitados dos direitos civis e critica o elevado poder desempenhado pelos médicos na definição e gestão do problema da incapacidade. Nesse contexto um argumento importante e atual reside na identificação da dependência aos especialistas que definem, interpretam e propõe estratégias de cura para as formas de incapacidade. De um modo geral se considera que os estudos funcionalistas mostraram-se insuficientes ao não reconhecerem adequadamente os fatores estruturais na origem dos fenômenos descritos (D'AVANÇO, 2000).

Já a matriz materialista prioriza a análise do conjunto da organização social que para funcionar se utiliza de uma lógica de discriminação daqueles que não respondem adequadamente às expectativas da ordem econômica. Nessa perspectiva, admite que a incapacidade é provocada e construída socialmente, sendo importante compreender os mecanismos responsáveis por este fenômeno, que também estão presentes na lógica de reconhecimento e resposta ao problema da incapacidade (D'AVANÇO, 2000).

Essa matriz se preocupa com a emancipação dos sujeitos em relação a esses mecanismos ao invés de defender apenas o direito à participação social, pois acredita que a sociedade tem, em primeiro lugar, que rever as regras de seu funcionamento para que possa existir espaço real para a diversidade e para as diferentes formas de existir dos sujeitos. As instituições responsáveis pela assistência também teriam que rever seus papéis, a partir do reconhecimento de sua função de controle social (D'AVANZO,2000).

Essas discussões são o pano de fundo dos debates que levaram a elaboração da CIF em substituição à classificação que a precedeu, a Classificação Internacional das Deficiências, Incapacidades e Desvantagens, a CIDID (FARIAS; BUCHALLA, 2005).

\section{A classificação de 1980}

Em 1976, a OMS aprovou a publicação de um instrumento de classificação que incorpora a perspectiva do modelo social, a Classificação Internacional das Deficiências, Incapacidades e Desvantagens (CIDID). Efetivamente lançada em 1980, a CIDID definiu a desabilidade ou incapacidade no contexto de um modelo linear que a coloca numa seqüência de níveis de experiência da saúde derivados de um estado patológico, ou de uma doença (Quadro 1). Dessa forma, uma modificação patológica, não necessariamente observável ou perceptível, pode comportar um dano da estrutura ou da função corporal:

QUADRO 1 - Modelo linear da CIDID

\section{Doença $\rightarrow$ Deficiência (Impairment) $\rightarrow$ Incapacidade (Disability) $\rightarrow$ Desvantagem (Handicap)}

Deficiência (dano ou déficit) é definida como qualquer perda ou anomalia (temporária ou permanente) das estruturas e funções psicológica, fisiológica ou anatômica e se constitui como desvio da norma biomédica, fundada em base estatística, possível de ser observada e mensurada. Se tal déficit implicar em dano para a pessoa que limite ou 
impeça o desenvolvimento de atividades ele determina uma incapacidade (OMS, 1989).

A incapacidade ou desabilidade (disability) é avaliada em seis áreas consideradas importantes para a reprodução social da pessoa: orientação, dependência física, mobilidade, trabalho e integração social e auto-suficiência econômica. Quando ocorrem conseqüências sociais negativas, isto é, perdas sociais nessas áreas, se constitui uma desvantagem (handicap), descrita como dano resultante de um déficit ou de uma desabilidade que limita ou impede o desenvolvimento do papel esperado para o sujeito que seria normal de acordo com dados do seu sexo, idade e contexto sócio cultural (OMS, 1989).

A noção de desvantagem (handicap) merece atenção, pois nela reside a tentativa de deslocar o problema da incapacidade, do indivíduo para seu contexto. Embora na CIDID a desvantagem (handicap) considere os limites sociais e ambientais que recaem sobre os sujeitos, sua colocação como terceiro elemento do esquema conceitual sugere que a desvantagem tem como causa a deficiência e a incapacidade (OMS, 1989).

Relembramos que, em sua origem, o conceito de handicap significa justamente o contrário do significado que possui no interior da CIDID. De acordo com Scuotto (2003) o termo: "nasce do sistema de penalização dos cavalos muito fortes para equilibrar a possibilidade de vitória dos concorrentes; por isso, conceitualmente faz referencia a uma desvantagem imposta na partida, para equilibrar a relação entre o indivíduo e o contexto, que coloca um limite para a capacidade de correr livremente" (p. 1).

A pessoa com desvantagem (handicap) é vista pela CIDID numa relação desfavorável com a sociedade, quando comparada às pessoas com capacidades íntegras, que a impede de desempenhar um papel social esperado pela sociedade e compatível com sua idade, sexo, condição social e cultural. Aqui se coloca a necessidade de discutir o conceito de norma subjacente à toda essa construção, pois reside nele uma das principais questões a serem enfrentadas se nosso objetivo estiver direcionado para a superação do conjunto de reduções operadas pelo modelo biomédico e suas implicações nos projetos assistenciais e de reabilitação (D’AVANÇO, 2000).

Como se vê, a estrutura proposta na classificação de 1980 compreende a incapacidade a partir de três conceitos de base conexos e hierarquizados -deficiência (impairment), incapacidade (disability) e desvantagem (handicap) - no qual o primeiro representa, em relação ao sucessivo, uma condição de possibilidade, e o sucessivo representa um reflexo social do precedente (Quadro 2).

QUADRO 2 - Adaptado de Scuotto, 2003

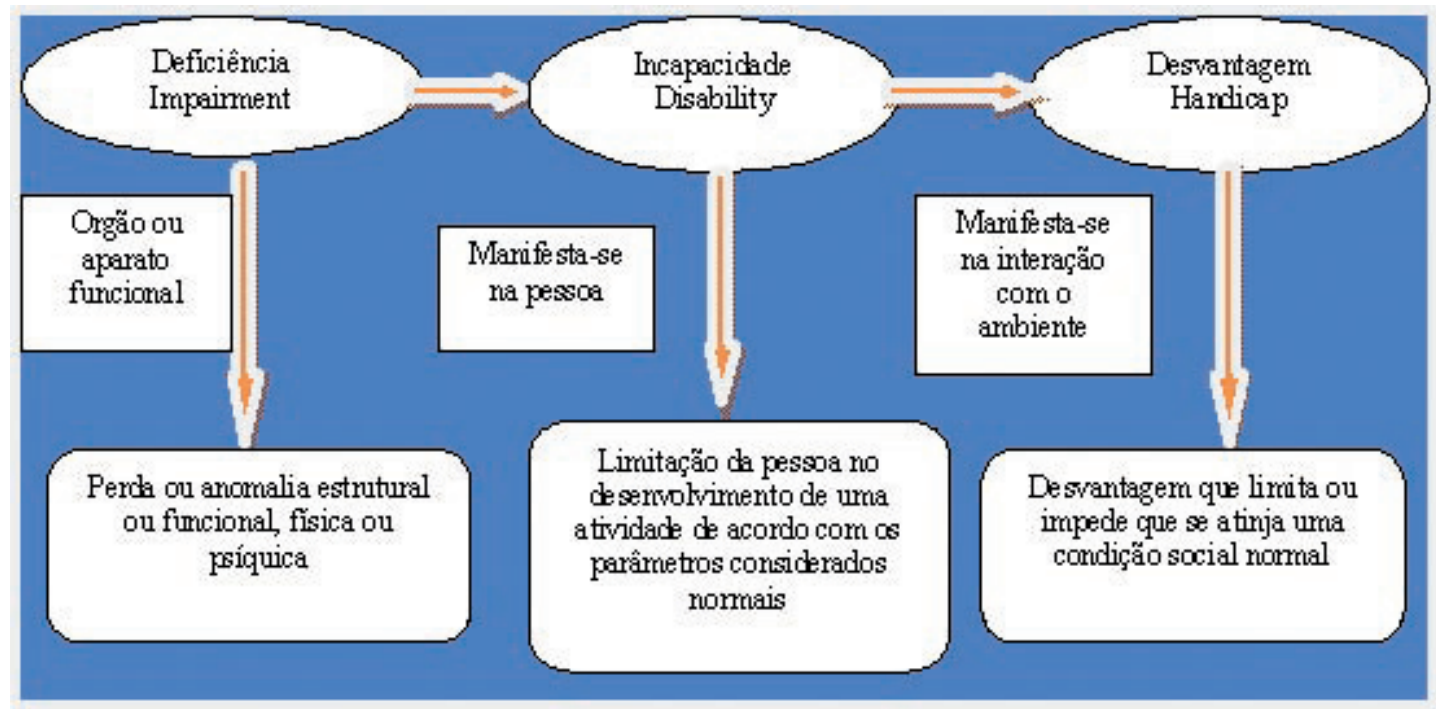

Embora admitindo a dimensão social das conseqüências da deficiência, a classificação de 1980 não consegue apreender a construção do processo de transformação da deficiência em incapacidade. $\mathrm{O}$ foco se detém no problema do funcionamento. A incapacidade é descrita como resultante de uma deficiência e a desvantagem como 
resultante de uma incapacidade, em uma perspectiva linear e causal que se apóia na compreensão biomédica do problema (D'AVANZO, 2000).

Esse modelo coloca no indivíduo a causa do problema e não leva em conta o papel estruturante do ambiente na compreensão da incapacidade e da desvantagem. Veremos que esta subordinação hierárquica entre os conceitos foi considerada incorreta, porque uma deficiência no funcionamento não produz necessariamente incapacidade ou obstáculo para a vida ativa. Incapacidade e barreiras para a vida social são, ao invés disso, os resultados de uma forma de acolhimento, ou a falta de, que o ambiente reserva para as pessoas com deficiência (D'AVANZO, 2000; IMRIE, 2004).

Este debate foi estimulado pela posição de alguns movimentos de pessoas com deficiência, com destaque ao papel da Union of the Physically Impaired Against Segregation (UPIAS), que desde 1976 já propunha um modelo alternativo que criticava o modelo biomédico e reivindicava a explicitação da importância do contexto social na produção da incapacidade. Disso resultou a segunda classificação fruto de um grupo de trabalho da OMS em 1997 (BICKENBACH et al., 1999).

Para a UPIAS a incapacidade deveria ser compreendida como restrição, criada socialmente, às pessoas com algum déficit, que limitasse a possibilidade de participação social igualitária. A incapacidade ou desabilidade, nessa perspectiva, é imposta ao sujeito por meio de barreiras objetivas, sociais e físicas. É a sociedade que desabilita o sujeito e sendo assim, é ela que deve remover barreiras e modificar seus padrões normativos. Esse debate tem a importância de politizar e desnaturalizar a compreensão sobre a desabilidade, reforçando ainda mais que esta não é um fenômeno individual e referido apenas à condição física ou mental dos sujeitos (BICKENBACH et al., 1999).

\section{O processo de transição: os pontos críticos da CIDID e os princípios da CIF}

Imrie (2004) discute que as teorias tradicionais sobre a incapacidade apontam para duas perspectivas distintas. A primeira, de caráter mais naturalista, tende a conceber mente, corpo e sociedade como "esferas separadas da existência humana" e deposita sobre o déficit ou deficiência, a raiz da incapacidade. A segunda perspectiva compreende a incapacidade como uma construção social, a partir da qual a sociedade oprime as pessoas incapazes. O autor afirma que ambas as perspectivas são falhas, na medida em que não reconhecem que o aspecto biológico e a sociedade compõem uma relação dialética.
Neste sentido, têm-se discutido cada vez mais a importância de se considerar a interação entre o corpo funcionalmente incapaz, as relações socioculturais e os processos que possibilitem a construção de uma compreensão da incapacidade que não seja reducionista. Atualmente, essa discussão surge com notável importância na CIF (OMS, 2001).

Retomando o debate já iniciado, a CIF substituiu a Classificação das Deficiências, Incapacidades e Desvantagens (CIDID), alvo de muitas críticas por seu caráter biomédico e por ser prioritariamente focado nas limitações individuais como principal fator determinante da incapacidade. Buscando afastar-se deste modelo, a CIF propõe que a incapacidade seja considerada na intersecção entre o corpo biológico e as estruturas sociais e institucionais. Define a incapacidade/desabilidade como um "fenômeno relacional por meio do qual as limitações funcionais de um déficit ou deficiência podem tornar-se incapacitantes devido a relações sociais mais amplas e atitudes" (IMRIE, 2004, p. 293).

Outra diferença significativa entre a CIF e a CIDID é a relação entre déficit/deficiência e doença. De acordo com a CIF, a presença de um déficit/deficiência "não indica necessariamente que há uma doença ou que o individuo deva ser considerado doente". Ao contrário, ela afirma que

"a questão (da incapacidade/desabilidade) é, portanto, atitudinal ou ideológica e requer mudanças sociais, as quais, em nível político, tornam-se questão de direitos humanos. A incapacidade/desabilidade torna-se, em resumo, uma questão política" (CIF, 2001; p. 25).

Essa mudança configura-se em importante proposta de transformação da compreensão que fazemos sobre a incapacidade e ganha ainda mais relevância se considerada a influência que as políticas propagadas pela OMS e os seus instrumentos causam na construção de políticas públicas em todo o mundo (IMRIE, 2004).

Conforme apontado por Bickenbach et al. (1999), diversos países basearam-se na CIDID para a construção de políticas e para a organização de seus sistemas de saúde.

Embora a CIDID tenha trazido contribuições significativas, em certo sentido ela reforçou o enfoque biomédico na compreensão da incapacidade/desabilidade, o que trouxe conseqüências para as estruturas, programas e políticas sociais e de saúde. A utilização deste instrumento, pelas políticas e programas de saúde, induz a compreensão de que a incapacidade e a desvantagem são fatores ocasionados por um déficit biológico e, por isso, requerem intervenção e tratamentos médicos. A CIDID também influenciou muitos países centrais a considerarem a adaptação e as estratégias individuais na forma de lidar com a incapacidade e no 
estabelecimento de prioridades para o desenvolvimento de políticas publicas, reforçando o foco da incapacidade/ desabilidade na pessoa. (IMRIE, 2004).

\section{A CIF}

A nova classificação, aprovada em maio de 2001, representa um instrumento elaborado para descrever e mensurar a saúde e a incapacidade. Se afirma como uma classificação dos componentes da saúde e não das conseqüências da doença, como a anterior e compõe a "família de classificações da OMS" que recomenda que sua utilização se dê de forma complementar, em conjunto com a CID-10 (Quadro 3) (OMS/OPAS, 2003).

QUADRO 3 - OMS (2003)

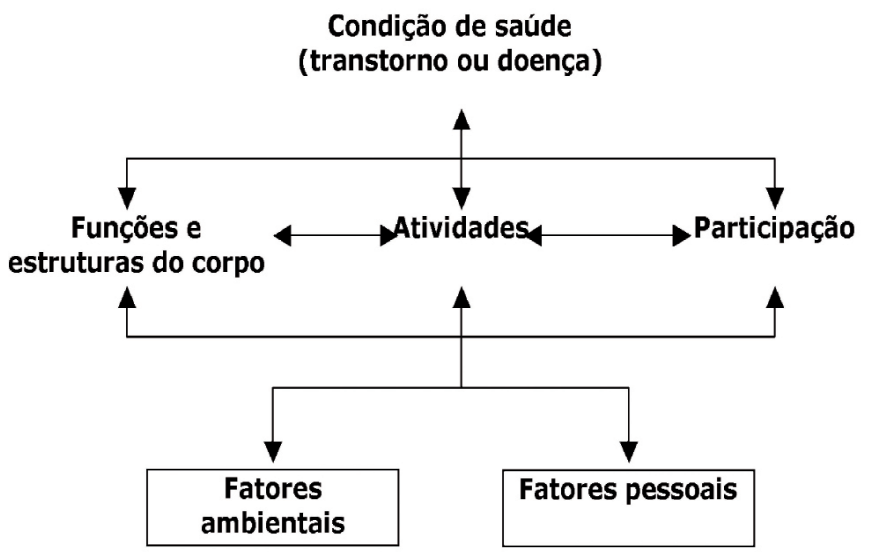

Baseada na abordagem biopsicossocial, a CIF se aplica de forma universal a todas as pessoas e não somente àquelas com algum déficit e busca romper com a seqüência linear e causal presente na CIDID. Pretende ainda ocupar uma posição de neutralidade etiológica que pressupõe a incapacidade/desabilidade como resultado de múltiplas determinações relacionadas ao contexto social onde a deficiência ou déficit não é considerado como causa necessária e/ou suficiente (D’AVANZO, 2000).

A CIF apresenta um conjunto de objetivos que podem ser assim sintetizados:

- Fornecer uma base científica para a compreensão e o estudo da saúde, das condições, conseqüências e causas que a determinam;

- Estabelecer uma linguagem comum para a descrição da saúde e de suas condições correlatas com o objetivo de melhorar a comunicação entre os diversos grupos que a utilizam, entre eles, os profissionais de saúde, os pesquisadores, os planejadores de políticas públicas, movimentos sociais e população em geral;

- Possibilitar o confronto entre dados coletados em diferentes países e em períodos distintos por pesquisadores, serviços e outras agências;
- Fornecer um esquema conceitual capaz de contribuir para a codificação e sistematização dos sistemas de informações em saúde.

Para alcançar esses objetivos, a OMS define algumas aplicações possíveis da CIF em três níveis: individual, institucional e social.

No nível individual:

- Para a avaliação de indivíduos: Qual o nível de funcionalidade da pessoa?

- Para o planejamento do tratamento individual: Que tratamento ou intervenções podem aumentar a funcionalidade?

- Para a avaliação do tratamento e outras intervenções: Quais são os resultados do tratamento? As intervenções foram úteis?

- Para a comunicação entre médicos, enfermeiros, fisioterapeutas, terapeutas ocupacionais e outros trabalhadores da saúde, do serviço social e agências comunitárias.

- Para auto-avaliação pelos usuários: Como eu poderia aumentar minha capacidade em mobilidade ou comunicação?

No nível institucional: 
MÂNGIA, E. F. et al. Classificação Internacional. CIF. Rev. Ter. Ocup. Univ. São Paulo, v. 19, n. 2, p. 121-130, maio/ago. 2008.

- Para propósitos educacionais e de treinamento.

- Para o planejamento e desenvolvimento de recursos: Quais serviços de saúde ou outros serviços serão necessários?

- Para melhorar a qualidade: Como atendemos nossos clientes? Que indicadores básicos para qualidade da atenção são válidos e confiáveis?

- Para gerenciamento e avaliação de resultados: $O s$ serviços que oferecemos são úteis?

- Para gerenciamento e avaliação de serviços e prestação de cuidados em saúde: Quão custo-efetivo são os serviços que oferecemos? Como o serviço pode ser aprimorado com melhores resultados a um baixo custo?

No nível social:

- Para critérios de elegibilidade para acesso a direitos tais como benefícios da seguridade social, pensões por incapacidade, seguro e compensação trabalhista: Os critérios de elegibilidade para acesso aos benefícios para incapacidades são baseados em evidências, apropriados aos objetivos sociais e justificáveis?

- Para o desenvolvimento de políticas sociais, incluindo revisões da legislação, o modelo da legislação, regulamentações e orientações, e definições para a legislação anti-discriminação: A garantia de direitos irá melhorar a funcionalidade no nivel social? É possivel mensurar essa melhora e ajustar nossas políticas e legislação de acordo com elas?

- Para avaliações de necessidades: Quais são as necessidades de pessoas com variados níveis de incapacidade, deficiências, limitações na realização de atividades e restrições na participação?

- Para avaliação do ambiente para implantação do desenho universal, acessibilidade, identificação de facilitadores ou barreiras ambientais e mudanças na política social: Como podemos tornar as estruturas e o ambiente social mais acessivel para todas as pessoas, aquelas com ou sem incapacidades? É possivel avaliar e mensurar as melhorias (WHO, 2002, p. 6).

\section{A base conceitual da CIF}

Mesmo reconhecendo que o desempenho de atividades é essencialmente relacional, pois se dá na relação com o mundo físico e deve ser contextualizado no ambiente, os terapeutas e os modelos de reabilitação avaliam a desabilidade em termos individuais. Se, ao invés disso, o ambiente fosse avaliado em termos das barreiras que oferece aos indivíduos, a causa da desvantagem seria nele situada e não mais na pessoa. Os ambientes onde ocorrem as relações expressam atitudes e valores socialmente construídos, assim, as características físicas, arquitetônicas e todo o conjunto de requisitos e habilidades práticas e subjetivas exigidos pela vida cotidiana estão norteados por um padrão normativo que, por si só, já pré seleciona aqueles que dele podem participar. Essa dinâmica contraria os princípios de igualdade e direitos postulados pela maior parte das sociedades contemporâneas (D'AVANZO, 2000; BICKENBACH et al.,1999; SARACENO, 1998).

A luta pelo reconhecimento dos direitos está presente nos movimentos sociais que passaram a pleitear o acesso e o gozo dos direitos de cidadania, dentre eles se destaca os movimentos das pessoas com deficiência e com transtornos mentais. Embora com características distintas, tais movimentos, identificados como "movimentos de minorias", têm em comum a estratégia da afirmação da diferença como marco para a reivindicação de direitos especiais (BICKENBACH et al., 1999).

Se, por um lado, essa estratégia levou a um amplo reconhecimento social sobre as questões e demandas desses grupos, gerando mudanças culturais, nas políticas sociais e legislações, por outro permitiu a critica aos limites dessa estratégia e ao fato dela gerar o risco do isolamento desses grupos, em identidades fixas e imutáveis (SARACENO, 1998). Nesse sentido, os defensores da perspectiva universalista discutem os limites dessa estratégia e avaliam que a falta de oportunidades e recursos não pode ser interpretada apenas como discriminação, mas como resultado de uma injusta distribuição de oportunidades e recursos sociais que limita todas as áreas da vida. Há na sociedade uma generalizada falta de reconhecimento das necessidades humanas, especialmente àquelas ligadas ao déficit, que resulta em perdas importantes na possibilidade de participação social de muitos segmentos da população (D’AVANZO, 2000; BICKENBACH et al., 1999).

Antes das necessidades assistenciais e específicas que, no geral, os terapeutas e os serviços de saúde reconhecem, há as necessidades básicas para a produção e reprodução de vida social e este é o ponto cada vez mais presente no debate quando se objetiva refletir sobre o tema da inclusão social. Nessa direção Zola (1989, p. 36) defende a "desmistificação da natureza específica da desabilidade" a adoção de uma estratégia universalista que reconheça que toda a sociedade está exposta ao risco da desabilidade/ incapacidade, dada a conexão entre doenças crônicas e desabilidade/incapacidade.

\footnotetext{
"Ver as pessoas com desabilidades como "diversas" e com necessidades, desejos e direitos "especiais" em um mundo como o nosso, no qual os recursos são em realidade finitos, as coloca em contraposição às necessidades, desejos e
} 
MÂNGIA, E. F. et al. Classificação Internacional. CIF. Rev. Ter. Ocup. Univ. São Paulo, v. 19, n. 2, p. 121-130, maio/ago. 2008.

direitos do resto da população" (ZOLA, 1989, p. 19).

O aumento da expectativa de vida propicia, por exemplo, que se insira em uma mesma agenda política os idosos e as pessoas com desabilidades. A idéia central seria a de não discriminar especificamente as pessoas com desabilidades como um grupo separado dos demais, pois os critérios que discriminam a desabilidade são políticos e não estatísticos.

Zola (1989) assinala que, talvez sem se dar conta, a abordagem das minorias tende a reforçar o ponto de vista médico sobre a desabilidade, como algo fixo e descontextualizado. Assim, em concordância com o modelo social da desabilidade/incapacidade, propõe que a desabilidade seja abordada como processual e contextual, conectada com uma estratégia que respeite as diferenças e amplie os limites da normalidade.

"A desabilidade não pode ser vista como um atributo que separa uma porção da humanidade de outra, mas uma característica variável, mas universal da condição humana, pois ninguém possui um repertório infinito de capacidades. Não há limites internos intrínsecos ao espectro de variação das capacidades humanas, entre habilidade e desabilidade há um continuum, e a total ausência de habilidades ou total ausência de desabilidades são situações puramente teóricas" (ZOLA, 1989 apud D’AVANZO, 2000, p.37).

\section{Considerações sobre a CIF}

Considerando a maneira pela qual a CIDID influenciou governos e a construção de seus sistemas de saúde e reabilitação, espera-se que a CIF também possa ser utilizada para transformar a compreensão sobre a incapacidade/ desabilidade e para moldar os futuros programas e políticas sociais e de saúde. No entanto, pouca ou nenhuma discussão tem sido realizada sobre as diretrizes conceituais e teóricas da CIF e em que grau tais diretrizes poderão contribuir para a construção de uma base coerente sobre a natureza da incapacidade/desabilidade e da deficiência na sociedade (IMRIE, 2004).

Com o objetivo de explorar este debate, apresentaremos as principais discussões apresentadas no artigo "Desmystifying disability: a review of the International Classification of Functioning, Disability and Health", de Imrie (2004).

$\mathrm{O}$ autor argumenta que alguns dos principais conceitos e princípios da CIF devem ser aprofundados e melhor justificados, a fim de evitar que a utilidade prática do instrumento fique limitada, sugerindo que tal aprofundamento também contribuiria para reduzir o grande número de diferenças interpretativas possibilitadas pela
CIF. Os elementos referidos pelo autor são: a definição de déficit/deficiência (impairment); a teoria biopsicosocial; e os princípios de universalização como base para os programas sociais e de saúde voltados para incapacidade/ desabilidade.

\section{- Definição de déficit/deficiência}

Imrie (2004), a partir de estudos de diversos autores, afirma que há uma lacuna nos estudos sobre incapacidade/ desabilidade, que diz respeito à definição sobre a natureza do déficit/deficiência. Podem ser encontradas diversas perspectivas, que vão desde a compreensão do déficit/ deficiência como uma diferença fixa e irredutível entre capazes e incapazes àquela que o concebe como uma diferença socialmente construída (THOMAS, 1998 apud IMRIE, 2004).

Entre esses dois pólos, é possível encontrar vários outros modelos que tendem ora para a perspectiva biológica, ora para a social.

A CIF, por sua vez, pretende localizar-se no contexto deste amplo leque, na medida em que considera que as limitações provocadas pelo déficit/deficiência estão ligadas às interações entre o aspecto biológico, fatores pessoais e limitações do ambiente. Entretanto, ao definir as funções e estruturas do corpo, a CIF tende a duas interpretações distintas: a primeira atribui ao déficit/deficiência um valor neutro, despersonalizado. Já a segunda declara a pessoa com deficiência como defeituosa, na medida em que define a deficiência como 'problemas na função ou estrutura do corpo, tais como desvio ou perda' quando comparados às normas estabelecidas estatisticamente, configurando-se, portanto, numa anormalidade. Assim, apesar da tentativa de introduzir um modelo complexo sobre a incapacidade, a CIF aborda a deficiência como "um fato", uma diferença biológica, corporal, pré-social (IMRIE, 2004, p. 294). Portanto, é possível verificar algumas incoerências no que concerne aos componentes Funções e Estruturas do corpo, especialmente no que diz respeito à natureza da deficiência. Tal aspecto, considerado negativo pela CIF, demanda maior aprofundamento e embasamento teórico para justificar o conceito de incapacidade adotado e para evitar desvios de interpretação e na utilização do instrumento.

\section{- Conteúdo da teoria biopsicossocial}

ACIF adota o modelo biopsicossocial como base teórica, compreendido como uma 'síntese das abordagens médica e social para definição da desabilidade/incapacidade' (BICKENBACH, 1999, p.1183). De acordo com Imrie (2004), a adoção dessa síntese tem como objetivo superar 
a hiper medicalização adotada pela CIDID e a tendência do modelo social em separar a desabilidade/incapacidade de suas origens médicas. Para a teoria biopsicossocial (BPS), os fatores determinantes para a função e a saúde são compreendidos nas dimensões biológica, pessoal ou psicológica e social, buscando desenvolver uma compreensão relacional ou não-dualística do corpo.

Imrie defende que a CIF não esclarece as origens teóricas ou o conteúdo do modelo BPS, assim como não apresenta a justificativa da adoção deste modelo como sua base conceitual. Isso acarreta em dificuldades para a construção de políticas públicas, para a utilização da CIF por profissionais de saúde e para pesquisadores. A CIF afirma que o objetivo da utilização da teoria BPS é 'oferecer uma sintese por meio da qual seja possivel elaborar uma visão coerente das diferentes dimensões da saúde, nos niveis biológico, individual e social' (ICF, 2001 apud IMRIE, 2004). Entretanto, não define a forma pela qual essa síntese pode influenciar a elaboração da 'visão coerente', na medida em que a CIF não apresenta a relevância da abordagem BPS no desenvolvimento de uma teoria da desabilidade/ incapacidade.

Para Imrie, quando se tenta buscar as origens da BPS, as ciências psiquiátricas aparecem como campo no qual se encontram suas raízes intelectuais (DILTS, 2001; SARAFINO, 2004 apud IMRIE, 2004). Neste sentido, as semelhanças entre a trajetória deste modelo no campo da saúde mental e, mais recentemente, na definição da desabilidade/incapacidade são evidentes. Mais uma vez, a preocupação em relação a esse limite da CIF é a lacuna que se abre para diversas possibilidades interpretativas, enquanto não houver a identificação das origens intelectuais da abordagem BPS e da justificativa e potencial contribuição na construção de uma teoria da desabilidade/incapacidade.

\section{- Natureza universal da desabilidade/ incapacidade}

A OMS afirma que "há um mal-entendido vastamente sustentado de que a CIF diz respeito apenas às pessoas com desabilidades/incapacidades; na verdade, ela diz respeito a todas as pessoas... em outras palavras, a CIF tem aplicação universal" (WHO, 2001, p.7). De fato, a CIF pretende oferecer um instrumento cuja aplicabilidade seja universal, tendo como base o conceito de universalidade de ZOLA (1989, p. 401), que argumenta que:

"uma abordagem da desabilidade/incapacidade referida exclusivamente às necessidades especiais é, inevitavelmente, uma abordagem de baixo alcance. O que nós precisamos são politicas mais universais" (ZOLA, 1989, p. 401; BICKENBACH et al., 1999).

Imrie (2004) defende que, apesar de valiosa, a opção da CIF em adotar a universalidade como base não é realmente discutida ou justificada. Para a CIF, a concepção de universalidade é baseada apenas no reconhecimento de que qualquer pessoa está vulnerável à aquisição de um déficit/ deficiência ou de doenças crônicas, que podem acarretar em desabilidade/incapacidade. Alguns autores argumentam que a doença é uma experiência de qualquer vida orgânica e que o curso natural da vida, ou o envelhecimento, aumentam inevitavelmente a proporção de pessoas com desabilidade/ incapacidade. Para Bickenbach et al. (1999, p. 1181), uma política universal procura desmistificar o caráter 'especial' da desabilidade, a partir do reconhecimento de que 'todas as pessoas têm necessidades que variam amplamente de formas mais ou menos previsiveis durante a vida'.

Segundo Imrie (2004), entretanto, o problema é que esse reconhecimento contrapõe duas posições, o universal e o particular. Para Zola (1989), uma política universal não poderia negar a importância de necessidades ou demandas específicas de pessoas com tipos particulares de deficiência, mas também não seria recomendável a criação do conceito universal da desabilidade/incapacidade com uma "estratégia adicional complementar" para desabilidades específicas. Isso implica na necessidade do desenvolvimento de "uma concepção de necessidades especiais que não seja baseada em quebrar as regras para alguns, mas em construir um mundo flexível para muitos" (p. 422).

Para Thompson e Hoggett (1996, p. 33, apud IMRIE, 2004), "a verdadeira questão da universalidade é estabelecer um padrão imparcial entre pessoas e grupos diferentes". Isso não impede a construção de políticas e programas de saúde seletivos ou 'especiais', ao contrário, tende a demonstrar um compromisso com a universalidade. O debate neste campo é como garantir que esses programas e políticas seletivos atuem efetivamente enquanto confirmadores da universalidade e não acabem, como frequentemente tem acontecido, como mais uma forma de estigmatizar e marcar pessoas e populações específicas (TIMUSS, 1976 apud IMRIE, 2004).

A partir deste artigo, Imrie (2004) procura destacar a importância da CIF na re-conceituação da desabilidade/ incapacidade e, especialmente, na transformação das formas da relação deficiência - doença - incapacidade e das estruturas, políticas e programas sociais e de saúde.

A maior contribuição da CIF, segundo o autor, é estimular a construção de uma compreensão relacional da 
desabilidade/incapacidade, na qual sejam considerados outros fatores determinantes: corpo, pessoa, contexto e fatores sociais e ambientais. Ao fazer isso, a CIF aponta que qualquer compreensão sobre funcionalidade e saúde deve necessariamente incorporar aspectos dos modelos biológico e social, o que representa seu compromisso com uma abordagem pluralística e consensual na construção de uma teoria sobre a incapacidade.

Para que isso seja possível, o autor defende que a natureza e a justificativa da utilização de alguns modelos teóricos adotados pela CIF necessitam de maior desenvolvimento, para que possam ser incorporados e utilizados tanto no campo prático como na construção de políticas e programas de saúde.

MÂNGIA, E. F.; MURAMOTO, M. T.; LANCMAN, S. The International Classification of Functioning, Disability and Health (ICF): elaboration process and debate about the disability question. Rev. Ter. Ocup. Univ. São Paulo, v. 19, n. 2, p. 121-130, maio/ago. 2008.

\begin{abstract}
The problem of disability has social and economic relevance to the most countries and dramatic proportions whether considering the appreciation of the existence of 600 millions of disabled people in the world. The World Health Organization (WHO) has systematically alerted the countries about the insufficiency of promoting programs of disabled people and underlies the necessity in comprehend this problem as a social responsibility question, especially concerning the guarantee of rights of people with disabilities. In this context, this paper intend to present the International Classification of Functioning, Disability and Health ICF - and some related discussions, as: the social construction of disability; the transition process between the ICIDH - International Classification of Impairments, Disabilities and Handcaps - and ICF; its conceptual basis and some theoretical considerations about the instrument. To achieve this, the paper explore especially three articles, whose reading we recommend: "La seconda classificazione internazionale di defcit, disabilitá e handicap dell'pms: confronto e integrazione di modelli di disabilitá", organized by Barbara D'Avanzo (2000), "Models of disablement, universalism and international classification of impairments, disabilities and handcaps", of Bickenbach and others (1999) and "Desmystifying disability: a review of the International Classification of Functioning, Disability and Health”, by Rob Imrie.
\end{abstract}

KEY WORDS: International Classification of Functioning, Disability and Health. Social responsability. Handicapped advocacy/legislation \& jurisprudence.

\title{
REFERÊNCIAS
}

BICKENBACH, J. E.; CHATTERJI, S.; BADLEY, E. M.; USTUN, T. B. Models of disablement, universalism and international classification of impairments, disabilities and handcaps. Social Sci. Med., v. 48, p. 1173-1187, 1999.

D'AVANZO, B. La seconda classificazione internazionale di defcit, disabilitá e handicap dell'OMS: confronto e integrazione di modelli di disabilitá, Lettera, Percorsi Bibliografici in Psichiatria, v. 26-27, april, 2000.

FARIAS, N.; BUCHALLA, C. M. A Classificação Internacional de Funcionalidade, Incapacidade e Saúde, Rev Bras Epidemiol., v. 8 , n. 2, p. 187-93, 2005.

IMRIE, R. Demystifying disability: a review of the International Classification of Functioning, Disability and Health. Sociol Health Ilness., v. 23, n. 3, p. 287-305, 2004.

OMS. Classificação Internacional das Deficiências, Incapacidades e Desvantagens (handicaps): um manual de classificação das conseqüencias das doenças. Lisboa, 1989.

OMS/OPAS. Relatório sobre a saúde no mundo - 2001. Saúde mental: nova concepção, nova esperança. Gráfica Brasil, 2001.
OMS/OPAS Classificação Internacional de Funcionalidade e Incapacidade e Saúde, CIF. São Paulo: EDUSP, 2003.

OMS/OPAS A incapacidade: Prevenção e reabilitação no contexto do direito de gozar o mais alto padrão possível de saúde física e mental e outros direitos relacionados. $47^{\circ}$ Conselho Diretor, $58^{\circ}$ sessão do comitê regional, Washington, D.C., EUA, 25 a 29 de setembro de 2006. Disponível em: http://www.paho.org/ Portuguese/GOV/CE/ce138.r11-p.pdf

SARACENO, B. Cidadania como forma de tolerância. Conferência. Rio de Janeiro, 1998. Disponível em: www.exclusion.net.

SCUOTTO, S. Il passaggio dalla Classificazione Internazionale delle Menomazioni, delle Disabilità e degli Handicap (I.C.I.D.H) alla Classificazione Internazionale del Funzionamento, della Disabilità e della Salute (I.C.F) dell'Organizzazione Mondiale della Sanità (OMS). Disponível em: http://www.lamaestra.it/s08/ downloads/approfondimento_ICF.pdf

WHO. Towards a common language for functioning, disability and health ICF. Geneva, 2002.

ZOLA, I. K. Toward the necessary universalizing of a disability policy. Milbank Quarterly, v. 83, n. 4, p. 1-27, 2005. 